\title{
Effect of 4-Day School District Closure on Influenza-Like IIIness Rates Among Students and Household Members - Kentucky, 2013
}

\author{
Elizabeth S. Russell*1,2, Yenlik Zheteyeva², Hongjiang Gao², Jeanette Rainey², Jianrong \\ Shi $^{2}$, Karen Wong ${ }^{2}$, Douglas Thoroughman ${ }^{1,2}$ and Amra Uzicanin ${ }^{2}$
}

${ }^{1}$ Division of Epidemiology and Health Planning, Kentucky Department for Public Health, Frankfort, KY, USA; ${ }^{2}$ Center for Disease

Control and Prevention, Atlanta, GA, USA

\section{Introduction}

Coordinated proactive school closures can help to reduce disease transmission in communities during an influenza pandemic; however, limited information is available about effects of school closures during influenza-like illness (ILI) outbreaks. A rural school district (District A) in Kentucky was closed during January 29-February 1, 2013, in response to an increase in ILI-related student absenteeism. We investigated effects of this closure by comparing self-reported illness among household members of students enrolled in District A with 2 adjacent districts (Districts B and C) that remained open during that period.

\section{Methods}

Teachers distributed paper surveys to all $533 \mathrm{~K}-12$ students enrolled in District A, and 1,113 in Districts B and C, combined. Surveys asked parents or guardians to identify household members who experienced ILI (defined as subjective fever and either cough or sore throat in the absence of a known cause other than influenza) during 3 periods, as follows: 2 weeks before closure, during closure, and 2 weeks after closure. Average daily ILI rates were calculated for each period by using generalized estimating equations for proportion of household members who experienced ILI during each period and dividing by the number of days included in that period. We used the difference-in-differences (DiD) method to compare ILI rate changes over time in District A with Districts B and C.

\section{Results}

Completed surveys from 100 households of District A students provided ILI data on 373 household members, including 175 students (32.8\% of district total). Surveys from 227 households of District B and $\mathrm{C}$ students provided comparison data for 894 household members, including 434 students (39.0\%). The average daily ILI rate for household members was $0.99 \%$ in District A during the 2 weeks before closure, $3.70 \%$ during the 4-day closure, and $0.70 \%$ during the 2 weeks after school closure; Districts B and C combined had an average daily ILI rate of $1.40 \%$ before, $3.67 \%$ during, and $0.86 \%$ after closure. The DiD of ILI rates from before to after the closure between District A and Districts B and C combined was $-0.25 \%(\mathrm{P}=.4)$.

\section{Conclusions}

The school closure in District A did not have an effect on ILI rates in student households, compared with districts that were not closed.

\section{Keywords}

Non-pharmaceutical Interventions; Influenza; School Closure 